ARTIGO

\title{
Los Servicios de atención en el periodo neonatal en América Latina
}

Consultor Regional Salud Materno infantil OPS. OMS.

\section{E. N. Suärez Ojeda *}

En este documento se aplica el instrumento conocido como Condiciones de Eficiencia de Servicios, a los servicios de Neonatologia de seis partes de América Latina. Además, se aplica una lista de preguntas especificas sobre aspectos de la relación Madre-Hijo que incluyó veinte de los servicios tomados con las Condiciones de Eficiencia.

Se concluye que la atención neonatal en $A$. Latina es dificitaria, ya que en promedio, está por debajo de la mitad de las condiciones aceptables del cuidado del Recién Nacido. Solo em dos de las seis partes los servicios sobrepasaban el 50\%. Los aspectos más deprimidos se identificaron en Educación para la Salud y Programación y Administración. En contra de lo frecuentemente expresado, los aspectos más costosos de la atención neonatal (Equipo y Planta Fisica) estaban mejor cubiertos. Por ello, se recomenda que el énfasis debe ponerse en el mejoramiento de organización y en la promoción de cambios de actitud del personal.

En cuanto a relación Madre-Hijo, se advirtió que la mayoria de los servicios no han incorporado principios básicos de la misma en las prácticas de atención perinatal.

\section{I - INTRODUCCIÓN}

Alrededor de 15 millones de nacimientos se producen en América Latina cada año. De ellos, un $60 \%$ o sea unos 9 millones, acontece en estabelecimientos 0 instituciones hospitalarios de la más diversa índole, desde enormes maternidades del sector público hasta pequeñas clínicas privadas, pasando por hospitales polivalentes, centros rurales, etc. El $40 \%$ restante, es decir unos seis millones de nacimientos, sucede en domicilio, atendido por parteras empíri. ca, algún agete de medicina tradicional o algún miembro de la propia familia, y con remotas posibilidades de acesso o servicios si surge alguna complicación para la mujer o el recién nacido. ${ }^{1}$

En la segunda mitad de este siglo se ha producido una 
tendencia incontenible hacia la institucionalización del naciniento y a la atención hospitalaria de la madre y el Recién Nacido en los primeros días del periodo postnatal. Junto a élla, se observa una fuerte medicalización del nacimiento, con llamativo aumento de las intervenciones sobre la madre, el recién nacido y sobre todo, la relación entre éllos y con su familia. Las motivaciones y recionalidad de este processo son difíciles de establecer, pero se los ha vinculado a la creciente urbanización, a la alfabetización y a la penetración de los medios masivos de comunicación, entre otros fenómenos concomitantes. Tampouco está claro todavía el efecto que estas tendencias pueden haber tenido en términos de morbi-mortalidad materna, neonatal e infantil. Es casi seguro que se han evitado muertes maternas por la pronta disponibilidad de sangre y equipos de internistas o de cirugía en el medio hospitalario, pero también se han generado otras con cesáreas innecesarias, realizadas en las condiciones precarias que prevalecen en la mayoría de los estabelecimientos. ${ }^{2}$ Basta recordar al respecto que cifras de 20 a $30 \%$ de cesáreas sobre el total de partos aparecen ya en varios paises de la region.

En cuanto a morbilidad y mortalidad neonatal no cabe duda que muchos recién nacidos de bajo peso se han salvado al nacer en servicios con Unidades de Cuidado Intensivo Neonatal y ya son varios los países de América Latina que muestran algún hospital de alta complejidad cuya tasa de mortalidad específica por peso de nacimiento se compara satisfactoriamente con las de países desarrollados. Por otro lado, estos servicios son la excepción y en esa compleja gama que forma la red asistencial de cada país, lo predominante son estabelecimientos de mediana y baja compleji. $\mathrm{dad}$, donde persisten prácticas como la separación de la madre y el recién nacido durante las primeras horas de vida, la inducción de la lactancia artificial, la prohibición de la presencia del marido durante el nacimiento, la restricción de las visitas de familiares, etc. Estas prácticas restrictivas han de tener, a no dudarlo, efectos ulteriores en el aumento de la morbilidad y la mortalidad neonatal e infaritil, especialmente si dentro de morbilidad incluimos la creciente patologia de tipo psicosocial (niño golpeado, niño abandonado, etc.)

Una vez producido el parto, lo normal y habitual es que la puerpera permanezca dos o tres días en el hospital, recibiendo cuidados mínimos y un examen rutirnario de alta. Luego del egreso, las cifras de control puerperal son sumamente bajas, oscilando alrededor de $15 \%$ del total de puérperas las que reciben algún cuidado profesional (médico, matrona o enfermera) como cifra subregional para América Latina. ${ }^{3}$ 
A pesar de éstas y numerosas otras deficiencias observadas, el fenómenos de hospitalización del nacimiento ha continuado y la demanda por camas de maternidad se hace sentir intensamente, con serias repercusiones econômicas y sociales para los países. Si se quisiera dar atención institucional al estilo y costos de tipo norteamericano a los 15 millones de nacimientos mencionados, habría que disponer de recursos adicionales del orden de los $50 \mathrm{mil}$ millones de dólares.

A esta demanda emergente de la población por camas hospitalarias, se agregan los costos y riesgos de una incorporación acrítica de tecnologías de alto costo, no siempre aplicadas por profesionales idóneos y a menudo prescriptas sin estricta justificación clínica.

Por todo ello parecío necessario lanzar una mirada crítica sobre los servicios de atención de salud maternoinfantil en general, abarcando el sector público oficial al que se tiene acceso como OPS y cuya responsabilidad alcanza a los grupos más necesitados de la población en la mayoría de los países. Dentro de los datos obtenidos sobre servicios de salud maternoinfantil, se analizarán en este trabajo los referidos a la atención neonatal en establecimientos hospitalarios de distintas dimensiones.

\section{II - VISIÓN GENERAL DE LOS SERVICIOS DE SALUD EN AMERICA LATINA}

Para una mejor comprensión de los datos que se van a presentar es conveniente describir, aunque sea brevemente, algunas características generales de los sistemas de salud y de los establecimientos que los integran.

Un primer hecho a mencionar es que estos paises tienen instalaciones hospitalarias y unidades de atención ambulatoria en importantes cantidades y en general distribuídas ampliamente en el territorio nacional. Por otra parte, el número de profesionales, particularmente de médicos, en relación a los habitantes es má que suficiente en casi todos los países, con tasas que varían entre un médico por cada 400 habitantes y $1 \mathrm{c} / 2,000 .{ }^{4}$ Lamentablemente, la eficiencia de estos establecimientos y la eficacia de estos profesionales en función de las necesidades de salud de la población es por lo menos cuestionable. No se ha podido encontrar algún tipo de relación significativa entre número de profesionales o número de camas de una especialidad con los indicadores de salud respectivos. Se ha reiterado en numerosos informes y estudios el bajo rendimiento de los equipos e instalaciones, la falta de coordinación entre los distintos niveles de atención, la escasa capacidad resolutiva del nivel primario, la inadecuada formación del médico para 
atender las patologías más frecuentes, etc. Pero subsiste el hecho de que los establecimientos están y le cuestan al erario público, los profesionales existen y constituyen importantes grupos de presion política y gremial y entre ambos insumen una porción nada despreciable del presupuesto oficial y del producto bruto nacional. Tal parece que todo mejoramiento de la salud y del proceso de su atención en la Region, debe tomar en cuenta esas presencias y tratar de ponerlas a funcionar adecuadamente $y$ en función de objetivos comunes de bienestar.

Todos los países de la subregion - con una sola excepción - tienen sistemas pluralísticos de atención, en los que se pueden identificar por lo menos tres subsectores:

a) El público $u$ oficial, frecuentemente atomizado en unidades de jurisdicción nacional, provincial, municipal, etc.

b) EI subsector Seguridad Social en franca expansion tanto em proporción de población cubierta, como en construcción de centros hospitalarios de alta complejidad. A su vez, este subsector suele dividirse según afiliaciones gremilaes, constituyendo microsectores, independientes entre sí y con escasa coordinación. En uno de los países llegan a contarse 380 microadministraciones de este tipo.

c) El subsector privado, con una amplísima gama de modalidades, desde la consulta individual de un profesional hasta grandes sanatorios que concentran alta tecnología, pasando por innúmeras formas y dimensiones de clínicas, consultorios, centros, etc.

Las relaciones entre estos subsectores son complejas y variadas. En los últimos años ha sido frecuente que la seguridad social actúe como financiador de servicios que proporcionan los establecimientos privados. Esto se ha asociado con aumentos del uso de tecnolgias y de intervenciones. En el caso particular de lo perinatal se asoció al incremento extraordinario en la tasa de cesáreas y al uso indiscriminado de la ecografía. Por otra parte, es muy frecuente que un mismo profesional comparta su tiempo laboral entre los tres subsectores, acrescentando la complejidad de las interre. laciones. No es raro que la unidad del sector público se transforme en punto de captación para la práctica privada y que ésta sea la vía real para acceder al uso de tecnolgias complejas que existen en los establecimientos públicos o de la seguridad.

Al no existir, en la mayoría de los casos, mecanismos explícitos de coordinación entre subsectores se promueven Cadernos de Saúde Pública, R.J., 4(3): 426-443, out/dez, 1987. 
estas vías espontáneas y se cae con frecuencia en la duplicación innecesaria de servicios, 'en el rechazo de pacientes que necessitam atención por no ser "Beneficiarios" o, paradójicamente, en la repetición de exámenes costosos y no exentos de riesgo.

Es en esta intricada maraña de oferta de servicios donde la mujer y su niño van a buscar atención para sus necesidades y es objetivo de este artículo tratar de ver, mediante la aplicación de algunos instrumentos de evaluación, en qué medida encuentran una respuesta adecuada en relación a principios y conocimientos actualmente aceptados.

\section{III - METODOLOGIA}

En este documento se presentarán observaciones basadas en la aplicación de encuestras evaluativas llamadas condiciones de eficiencia, de amplia difusión en las Américas. ${ }^{5}$

Estas encuestas son listas de cotejo en ląs que se establecen los requisitos que un servicio debiera llenar para brindar una atención de salud acorde con los conocimientos y tecnologías aceptadas. El cumplimiento de cada uno de esos requisitos otorga un porcentaje, cuyo valor se estableció en consulta con expertos en el tema (sistema DELPHI). La suma de todos los puntos correspondientes a respuestas positivas da el puntaje total del servicio que, expresado en porcentaje, estaría indicando en qué proporción el mismo reủne las condiciones para una atención satisfactoria. ${ }^{6}$

Las preguntas se agrupan en áreas temáticas o "rubros", tales como:

Planta Física

Recursos Materiales

Recursos Humanos

Normas y Procedimientos

Programación y Administración

Suministros

Educación para la Salud

En este caso se aplicó la encuesta para servicios de Neo. natología (Anexo No. 1). Los aspectos psicológicos y socia. les (relación madre-hijo, participación de la familia, promoción de la lactancia materna) están considerados en los rubros referidos como Normas y Procedimientos y Educación para la Salud. Además, se incorporó un cuestinario específi. co que se incluye commo Anexo No. 2.

En cada pais se hace la discusión del modelo propuesto y se introducen las modificaciones necesarias para sua edecuación, de modo que al evaluar los servicios se está comparando con criterios aceptados por los propios professionates del pais. 
Em este trabajo se analizarán datos obtenidos con la aplicación de estas encuestas en seis países de América Latina en el periodo 1980-85. La muestra abarca servicios de atención neonatal (Neonatología) en hospitales del sector público, de paises con sistemas polivalentes de servicios de salud. Del total de servicios encuestados, se tomaron aquellos que incluían más de 1,000 nacimientos anuales y que corresponden a cabeceras de área o región de salud. En cada país de los considerados se incluyó un servicio de alta complejidad para el país, instalado en área metropolitana.

En los países mayores por extensión geográfica y población la colección de datos se hizo por Estado e Provincia $y$ en algunos casos abarcó solo uno de éllos. En esta ocasión se analizarán datos de 45 hospitales, distribuidos en los distintos paises y representando diferentes niveles de complejidad hospitalaria según lo expresado antes.

Los valores obtenidos se consolidarán en los diferentes rubros (Planta Física, Educación Sanitaria, etc.) obteniendo valores globales para todos los paises incluidos. Luego se desglosarán por país haciendo comparaciones entre ellos y se tratará de caracterizar tipos de atención según población atendida (por ej. población metropolitana vs. resto de país).

Por la forma de colectar los datos, esto no es una muestra representativa de la atención neonatal en América Latina, pero porporciona una visión general de la manera en que se está atendiendo al Recién Nacido y su madre en los Hospitales de esta parte del mundo.

En 1985, la OPS reunió un grupo de expertos de varios países de la Región, que produjeron una serie de guías para todos los servicios de atención de la mujer y el ninõ, inclu. yendo lso de Neonatología. Estas guías se están aplicando de manera sistemática en los países de América Latina y permitirán tener en el futuro un diagnósíico más aproximado de las realidades asistenciales en los establecimientos de salud de las Américas. ${ }^{7}$

\section{IV - RESULTADOS Y COMENTARIOS}

Los servicios encuestados en el transcurso de esta década, aplicando parámetros de medición en cada caso ajustados a la realidad y opinión profesional, revelan, en resumen, un valor global( $46,8 \%)$, que señala numerosas falencias. La cifra de 46,8 señala que menos de la mitad de los requisitos y condiciones deseables para un buen cuidado neonatal se están cumpliendo en los servicios. A nuestro juicio, ésta impresión numérica está mostrando um hecho cierto observable a lo largo de América Latina y el Caribe. Los servicios de atención neonatal adolecen de serias deficiencias en casi todos sus componentes. 
Los datos presentados en la Tabla No. 1 muestran además que en sólo 2 países de los 6 considerados, los servicios de Neonatología estaban por encima de la mitad de lo requerido. Particularmente, crítica es la situación de aquel. los que no alcanzaron siquiera $40 \%(37,1$ y 34,5$)$. Aún los que lograron mejores valores, no pasaron del $60 \%$. Cabe observar que en varios de los países encuestados se encontró algún servicio de alto nivel (alrededor de $80 \%$ ), pero ésta alta valuación se diluyó luego al promediarse con los demás servicios incluidos en la observación.

Contrariamente a la frecuente expresión de pobreza o carencia de equipos y bienes físicos, se advirtiró que en su conjunto los servicios están mejor en los aspectos relacionados a "Planta Física" (edificio, tipo de material de la construcción, instalaciones de oxígeno y aspiración, espacios para los profesionales) y Recursos Materiales (equipamiento en incubadoras, respiradores, olímetros, etc.). La verda. dera área crítica está en la Programación y Administración del Servicio (39\%). Perguntas a la organización, al conocimiento de indicadores básicos de la problación atendida, a la coordinación con la atención ambulatoria y a los servicios de apoyo, reciben con frecuencia respuestas negativas. Esta falencia en aspectos esenciales de la administración de los recursos hacen dudar de la utilidad de poner más fondos en establecimientos de tan escasa eficiencia. En otras palavras, parece estéril un aumento de presupuesto que no se acompañe o sea precedido por una revisión de la concepción orgánica de estos servicios y un adiestramien to básico del personal de conducción en Gerencia y Administración Hospitalaria. El desinterés del personal intrahospitalario por los indicadores básicos de salud de la población del área que rodea al Hospital revela el divorcio y contradicción existente entre lo clinico-asistencial y lo preventivo-sanitario. Esto ha llevado a observar costosas unidades de Cuidado Intensivo Neonatal, como en lugares donde aún ocurren muchas muertes por tétanos del recién nacido.

Aún más deficitario en su valor numérico, pero respondiendo a la misma concepción de Atención Médica Reparativa, es el rubro Educación Sanitaria (34\%). Actividades essenciales y de alto rendimien to en los países en desarrollo, como las charlas y contactos educativos con las madres en su paso por los servicios o la promoción de la lactancia materna, son frecuentemente olvidadas o reciben poca atención.

En orden ascendente siguen los rubros referentes a "Suministros" (43\%) y "Normas y Procedimientos" (50\%). El primero muestra uno de los defectos crónicos de los sistemas de salud de esta parte del mundo: luego de un costoso esfuerzo para construir y equipar un establecimiento, no se le proveen regularmente insumos esenciales para su fun- 
clonamiento y mucho menos se aplican mecanismus ue iriantenimiento preventivo de los equipos. El resultado es que los beneficios que la comunidad aguarda de estas inversiones, sólo se reciben de manera discontínua y parcial.

El componente de "Normas y Procedimientos" alcanza un nivel menos bajo de lo esperado, cumpliéndose la mitad de las especificaciones observadas (50\%). Este valor es más alto que lo habitualmente observado en Servicios de Obstetricia y Pediatría. Talvez, la Neonatología como disciplina de desarrollo más reciente, sea algo más respetuosa de normas y procedimientos y no impere el individualismo médico $\tan$ difundido en otras especialidades.

La observación reiterada de poca aplicación de principios básicos de relación madre-hijo y presencia familiar en el nacimiento y período postnatal inmediato, nos sugirió utilizar un cuestionario básico sobre el tema, a fin de profundizar el conocimiento de la situación. El mismo consta de 12 preguntas y observaciones que se formulan a personal del servi. cio, se observan directamente o se pregunta a las madres y familiares. El mismo se adjunta como Anexo No. 2 y fue aplicado en 20 servicios de cinco países de América Latina.

De manera general se advirtió un fuerte predominio de las respuestas y observaciones negativas. De las 12 preguntas, sólo en una prevalecieron las respuestas realmente afirmativas. Es la que se refiere a la práctica de alojamiento conjunto (pregunta No. 3). En las demás hubo siempre dominio de las negativas y en algunos casos se detectaron sensibles diferencias entre lo informado por distintos miembros del personal y por las madres. Tabla No. 2.

Los aspectos menos aceptados en la práctica son: acceso de los padres a la Unidad de Cuidado Intensivo Neonatal y presen cia del padre en Sala de Dilatación y/o de Expulsión. También se advirtió resistencia a las visitas de otros hijos de la pareja durante la hospitalización de la madre y el recién nacido.

En resumen, la incorporación de estos contenidos básicos de psicología neonatal familiar es todavía muy restringida y los servicios muestran un elevado grado de rigidez y predominio de criterios quirúrgicos en cuanto al cuidado deo nacimiento y el recién nacido.

\section{V - REFLEXIÓN FINAL}

Esta observación de servicios de atención neonatal no permite hacer conclusiones terminantes por su característica amuestral. Sin embargo, de una visıón general de cómo se cuida al recién nacido y su madre en las condiciones prevalentes da América Latina. 
De una manera global podemos decir que dichos cuidados son insatisfactorios y que cumlen menos de la mitad de las condiciones que debieran tener según los propios integrantes de la oferta. Estas áreas críticas son más marcadas en lo que podemos llamar "componentes blandos" (software) tales como Administración, Normatización, Educación Sanitaria y Aspectos Psicológicos. En contra de lo expresado muchas veces, la existencia de facilidades edilicias o de equipos de alta tecnología no es $\epsilon 1$ recurso limitante. Las notables diferencias en mortalidad neonatal no se explican tanto por disponer de más o menos aparatos, sino por la manera en que se organizan los recursos materiales y humanos y, en gran parte, por las actitudes del cuerpo profesional y el equipo de salud en relación a la comunidad a la que sirven.

A pesar de este panorama, por cierto nada atractivo, el fenómeno concreto es que la población afluye cada vez más a estos servicios y presiona por su expansión y fortalecimiento. Por otra parte, estos establecimientos están presentes a lo largo de casi toda América Latina, con sus edificios, sus equipos y su personal que constituye un componente importante de la fuerza laboral y política de los paises. En ésta Región al menos, parece que para mejorar las condiciones de salud materna y neonatal, no queda otro camino que corregir y fortalecer los servicios de salud existentes. Pero ello deber ser precedido de un replanteo, doctrinario en cuanto a su finalidad y destino, de una re-educación de su personal, en especial en cuanto a actitudes y aspectos éticos y de una revisión exhaustiva del sistema de salud de cada país.

La aplicación de estos modestos instrumentos de observación permitió percibir, además de las restricciones y debilidades, la posibilidad de recuperación de la eficiencia y calidad de la atención neonatal actuando sobre áreas críticas de tecnología de processo: mejor administración, re-educación del personal y los usuarios, actualización de normas y procedimientos. 
TABLA No 1

CONDICIONES DE EFICIENCIA - SERVICIOS DE NEONATOLOGIÄ VALORES PORCENTUALES POR RUBRO Y POR PAIŚ. AMERICA LATINA*

Pais/ Pl. Rec. Recursos Normas y Progr.y Suminis- Educ. Global rubro Física Materiales Humanos Procedimientos Adm. tros Salud

\begin{tabular}{|c|c|c|c|c|c|c|c|c|}
\hline A & 39 & 33 & 64 & 51 & 20 & 33 & 20 & 37.1 \\
\hline B & 49 & 55 & 47 & 65 & 46 & 45 & 37 & 49.1 \\
\hline $\mathrm{C}$ & 67 & 65 & 58 & 42 & 33 & 35 & 31 & 47.2 \\
\hline D & 61 & 75 & 43 & 66 & 58 & 63 & 45 & 58.7 \\
\hline $\mathrm{E}$ & 48 & 45 & 26 & 31 & 33 & 29 & 30 & 34.5 \\
\hline $\mathrm{F}$ & 69 & 72 & 61 & 46 & 41 & 53 & 39 & 54.4 \\
\hline \multicolumn{9}{|c|}{ RESUMEN } \\
\hline & 56 & 58 & 50 & 50 & 39 & 43 & 34 & 46.8 \\
\hline
\end{tabular}

* Datos obtenidos de la aplicación de Esquemas de Evaluación de Condiciones de Eficiencia, $1980 / 85$.

TABLA No 2

PREGUNTAS REFERIDAS AO APEGO Y ASPECTOS PSICOLOGGICOS DE LA ATENCION NEONATAL - AMERICA LATINA 1984/86

\begin{tabular}{ccccc}
\hline Pregunta* & Negativa & Afirmativa & Incierta y Variable & Total \\
\hline 1 & 8 & 6 & 6 & 20 \\
2 & 9 & 6 & 5 & 20 \\
3 & 7 & 13 & - & 20 \\
4 & 12 & 8 & - & 20 \\
5 & 12 & 4 & 4 & 20 \\
6 & 9 & 5 & 6 & 20 \\
7 & 15 & 5 & 1 & 20 \\
8 & 12 & 7 & 4 & 20 \\
9 & 9 & 5 & - & 20 \\
10 & 15 & 6 & 3 & 20 \\
11 & 11 & 1 & 2 & 20 \\
12 & 17 & 5 & &
\end{tabular}

* Ver contenido de cada pregunta en Anexo No. 2.

Cadernos de Saúde Pública, R.J., 4(3): 426-443, out/dez, 1987. 


\section{GRÁFICO № 1}

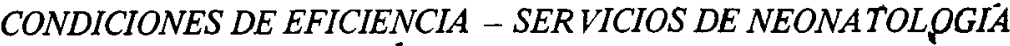 PAISES DE A. LATINA}

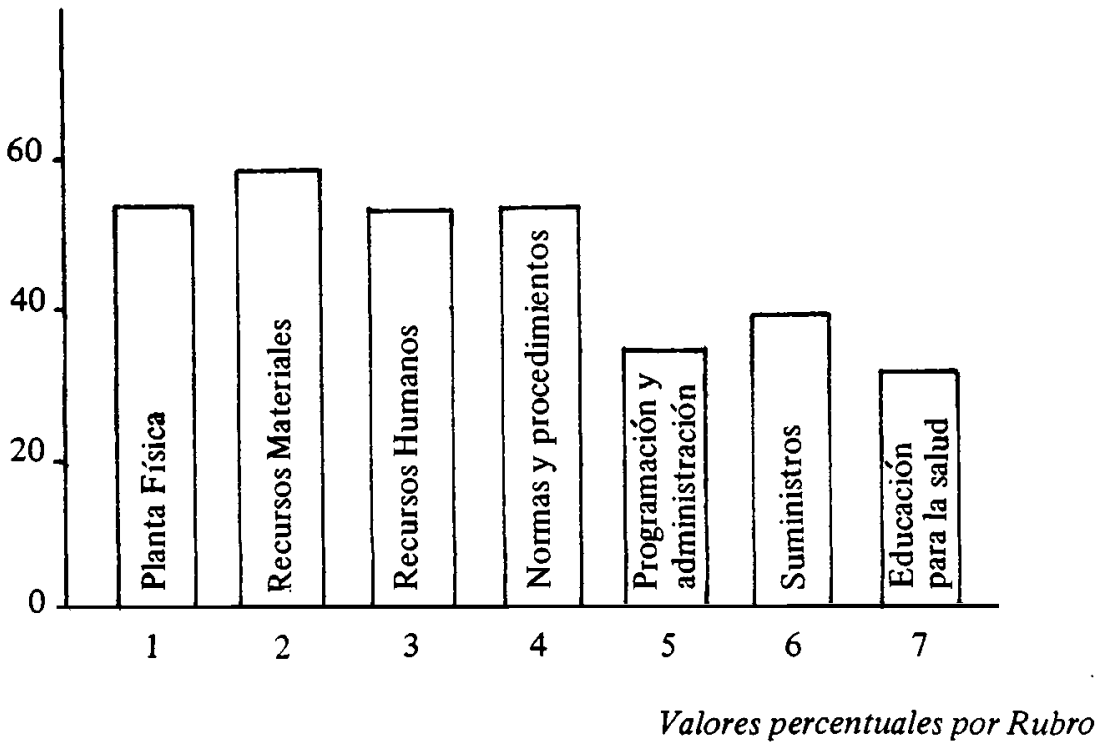

11111111111111111111111111111111111111111111111111111111

The thecnique known as Services'Efficiency Conditions is employed to Neo Natal Services in six areas of Latin America. Furthermore, a ist of specific questions about mother and child relationships is applied to twenty of the forementioned services.

Neo Natal Care in Latin America is regarded as deficient, considering that, on average, the acceptable conditions for Newborn's Care is under 50\%. This level is only exceed in two out of the six areas.

The most depressed items are Health Education and Programming and Administration. Contrary to what is commoonly expressed, the most expensive costs of Neo Natal Care (Equipment and Physical Plant) were better covered. Therefore, it is recommended that the emphasis should be put on organizational improvement and attitudinal changes of the staff.

Regarding to Mother and Child relationship, it is warned that most of the services have not embodied basic principles about it in their practices of Perinatal Care. 


\section{REFERENCIAS BIBLIOGRÁFICAS}

1. Coverage of Maternity Care - WHO/FHE/1985 - GENEVAFHE/85.1

2. Estudio y Preyención de la Mortalidad Materna en las Américas - OPS. Documento Preliminar Washington, D. C., 1986.

3. Evaluación del Plan Decenal de Salud - OPS, Washington, D. C., 1981.

4. Hospitales en las Américas. Publicación Científica No. 416, OPS, Washington, D. C., 1981.

5. Condiciones de Eficiencia de los Servicios de Atención Maternoinfantil. N. Baranchuk, E. N. Suárez Ojeda, Ediciones SMI, Buenos Aires, 1971.

6. Condiciones de Eficiencia de los Servicios Maternoinfantil, OPS, Washington, D. C., 1985. HPM/HSD.

7. Evaluación y Organización de Servicios de Salud para la Atención Primaria Maternoinfantil, Fascículo I, G. A. O. Alleyne, Elsa Moreno y colaboradores, OPS, Washington, D. C., 1985.

8. Evaluación de Tecnología - PNSP/85/30/25, OPS, Washington, D. C., 1985, Frenk J y Peña J.

9. Strategy for Evaluating Health Services, Institute of Medicine, National Academy of Sciences, Washington, D. C. , 1973. Kessner D. M. et al.

10. Donabedian, A. Quality Assessment and Monitoring: Retrospect and Prospect - Evaluation and the Health Professions. 6 (3) 1983.

11. Hinn, L. S. Factors Associated eith Patient Evaluation of Health Care, Milbank Memorial Fund, 1975.

12. Condiciones de Eficiencia de los Servicios Maternoinfantiles. PESMIB. N. Chang, N. Suárez Ojeda y colaboradores, 1974, Santiago, Chile.

13. Condiciones de Eficiencia de los Servicios de Salud Maternoinfantiles, Ministerio de Sanidad, Venezuela, 1985.

14. Niveles de Complejidad y Condiciones de Eficiencia, Servicios de Salud Maternoinfantiles, Honduras, 1985.

15. Evaluación de Eficiencia de los Servicios Maternoinfantiles en el Valle del Cauca J. Rico Velasco y colaboradores, 1985. Cali, Colombia.

16. Evaluación de los Servicios de Atención Médica, N. Baranchuk, M. D., M. Vázquez Vigo y colaboradores, Buenos Aires, Argentina, 1985.

17. Calidad de Atención Médica en Internación, L. P. Borini, N. F. Busso, $y$ colaboradores, Buenos Aires, Argentina, 1985.

18. Quality Assurance of Health Services, Public Health in Europe No. 16 - EURO, Copenhagen, 1982. 


\author{
ANEXO № 1 \\ ESQUEMA DE EVALUACIONN DE SER VICIOS \\ DE NEONATOLOGIÁ \\ IDENTIFICACIÖN DEL ESTABLECIMIENTO
}

Nombre:

Región:

Estado y Provincia:

Localidad:

Dirección:

Fecha:

\title{
RUBROS O CATEGORÍAS PARA SERVICIOS
} DE NEONATOLOGİA

\author{
I PLANTA FISICA \\ II RECURSOS MATERIALES \\ III RECURSOS HUMANOS \\ IV NORMAS Y PROCEDIMIENTOS \\ V PROGRAMACIÓN Y ADMINISTRACIĆN \\ VI SUMINISTROS \\ VII EDUCACIÓN PARA LA SALUD
}

\section{SERVICIOS DE NEONATOLOGIA}

\section{I - PLANTA FIŚICA}

1. La maternidad y la unidad de recién nacidos estan separadas del tránsito del resto del Hospital.

2. Hay facilidades físicas para alojamiento conjunto 10

3. Hay piso y paredes lavables 5

4. Hay espacio para atención inmediata del recién nacido

5. Hay $\mathrm{O}_{2}, y$ aspiración central provistos por tubería tanto en la sala de partos, como de puerperio y de recién nacidos.

6. Hay aire comprimido central provisto por tubería tanto en la sala de partos, cómo de puerperio y de recién nacidos.

7. Hay por lo menos $2.8 \mathrm{~m}^{2}$ por cuna

8. Cada sala tiene lavatorio accionable con el codo, pie o rodilla

9. Hay sala de observación durante el período de adaptación del recién nacido. 
10. Hay sala de observación para los sospechosos de infección.

11. La iluminación natural es suficiente para las actividades diurnas corrientes.

12. Posee oficina para médico, enfermera o matrona

13. Existe estación de Enfermería ubicada correctamente.

14. Existe sistema de una via para circulación de ropa sucia.

15. Existe sala de procedimiento.

16. La mitad de las paredes es de material transparente

17. Existe área de lavado y desinfección de materiales

18. Existe un sistema para regular la temperatura ambiental.

19. Existe un WC para uso exclusivo de los miembros del personal.

\section{II - RECURSOS MATERIALES}

1. Hay en cada sala, recipientes cubiertos para ropa, pañales sucios y materiales de desechos.

2. Hay una balanza por cada sala.

3. Hay un infantómetro.

4. Hay cinta metálica inextensible

5. Hay bandejas con equipo de atención individual para cada niño con termómetro, bajalenguas, etc.

6. La unidad tiene refrigerador para su uso exclusivo (substancias biológicas, leche, etc.)

7. Hay por lo menos 2 incubadoras en buen estado de funcionamiento por cada 1.00 nacidos vivos.

8. Las incubadoras son todas de circuito cerrado 8.1 solo el $50 \%$.

9. Hay equipos de luz para foteterapia.

10. Hay cunas calefaccionadas para tratamiento además de las de dotación.

11. Hay equipo parà intubación y reanimación según normas.

12. Existe equipo para termoregulación (cúpula térmica).

13. Se dispone de una incubadora de transporte por 3.000 nacidos vivos.

14. Los colchones y almohadas son lavables y esterilizables

15. Hay equipo para febloclises y punción lumbar

16. Hay un estetoscopio para cada sala de atención

17. Hay oftalmoscopio

18. Existe equipo adecuado para ex-sanguineo-transfusión de sangre. 
19. Se dispone de oxímetro. 5

20. Existe equipo de humidificación y calentamiento $\mathrm{de} \mathrm{O}_{2}$

21. Existem respiradores mecánicos (uno por cada 3.000 nacidos vivos).

22. Hay equipo para el estudio de equilibrio ảcido bases en las 24 horas.

23. Hay bomba para extracción de leche.

24. Dispone de equipo audiovisual.

25. El servicio dispone de efectiva mantención y reparación de los.aparatos.

\section{III - RECURSOS HUMANOS}

1. Se ha nombrado un médico jefe de unidad, pediatra con especialidad en recién nacidos.

1.1 Es pediatra sin especialidad en recién nacidos

2. Tiene capacitación en administración y organiza. ción de servicios de salud.

3. Hay pediatra permanente las 24 horas. 3.1 Hay pediatras de llamada.

4. Hay residencia pediátrica con rotación en neonatología.

5. Hay una hora médica por cada seis recién nacidos normales.

6. Hay una hora médica por cada cuatro recién nacidos patológicos.

7. Hay una enfermera jefe exclusiva de la Unidad.

8. Tiene capacitación en atención de recién nacidos en centros docentes reconocidos.

9. Hay enfermeras las 24 horas del día.

10. Hay una auxiliar por cinco cunas de recién nacjdos normales por las 24 horas.

11. Hay una auxiliar por tres cunas de recién nacidos patológicos por 24 horas.

12. El personal auxiliar recibe adiestramiento en servicios, con atención de recién nacidos de acuerdo con las necesidades locales.

13. Tiene secretaria de servicios.

\section{IV - NORMAS Y PROCEDIMIENTOS}

1. Está especificado por escrito el plan de actividades diarias de médicos, enfermeras y auxiliares. 1.1 Solo de Enfermeras.

2. Hay un pediatra en todos los partos.

2.1 Hay un pediatra solo en los partos provenientes del embarazo de alto riesgo. 
3. Se mantiene siempre lista una incubadora o cuna calefaccionada en sala de atención.

4. Se realiza la reanimación del recién nacido con técnica adecuada.

5. Se mide APGAR en sala de partos.

6. Se realiza profilaxis de la infección ocular. 10

7. Se efectúa pesaje del Recién Nacido. 15

8. Se efectúa medición de la talla. $\quad 10$

9. Se efectúa medición del perímetro craneano. 15

10. Se utiliza gráfica de crecimiento intra-uterino (pe. so/edad gestacional).

11. Se evalúa el grado de madurez del recién nacido (Capurro, Dubowitz, etc.).

12. Se efectúa identificación del recién nacido en sala de parto.

13. Hay normas escritas de atención para los recién nacidos normales.

14. Hay normas escritas de atención para las principales patologías (recién nacido pretérmino, bajo peso para la edad gestacional, ictericia, etc.)

15. Antes de Examinar al recién nacido se efectúa lavado de manos con agua y jabón según normas.

16. Se aplica vacuna $B C G$ al recién nacido normal.

17. Los recién nacidos infectados son tratados con técnicas de aislamiento.

18. I a historia clínica y los demás documentos, se escriben fuera de la sala de hospitalización.

19. Se lavan piso y paredes según normas.

20. Se utilizan microtécnicas para exámenes de laboratorio.

21. Se cumplen las normas de esterilización de equipos e instrumental.

22. Hay desinfección terminal de la incubadora, cuna y transportadora.

23. A todo el personal se le practica un examen médi. co y bactereológico de admisión.

24. El mismo se repite cada seis meses y despúes de licencia por enfermedad infecciosa.

25. En caso de brote epidémico se somete al personal a una investigación epidemiológica.

26. Se mantiene una ficha de salud de cada funcionario.

27. Se lleva un documento clínico individual por cada recien nacido donde se efectúa y se registra el examen médico completo a sua ingreso a la unidad y se anota su evolución y exámenes complementa. rios ( $\mathrm{HC}$ ).

28. Existe registro para todos los nacimientos (nacidos vivos y mortinatos).

Cadernos de Saúde Pública, R.J., 4(3): 426-443, out/dez, 1987. 
29. En toda $\mathrm{HC}$ se escribe un resumen o epicrisis al dar el alta.

30. Se aplican técnicas asépticas normatizadas en la preparación de alimentos y su traslado.

31. Se aplican técnicas asépticas normatizadas en la recolección y manutención de leche humana.

32. Se realizan autopsias sistemáticas según normas. 10

33. Se efectúa auditoría de los fallecidos. 10

\section{V - PROGRAMACIÓN Y ADMINISTRACIÓN}

1. El servicio de neonatología está organizado según programa.

2. Se conoce la población del área de atención del establecimiento.

3. Se conoce la tasa de mortalidad de dicha población. 10

4. Se conoce la tasa de mortalidad perinatal e infantil.

5. Se conoce la mortalidad según peso de nacimiento. 10

6. Existe un sistema de coordinación con el servicio de obstetricia.

7. Existe un sistema de coordinación con el servicio de Pediatría.

8. Existe coordinación con el Registro Civil.

9. Hay egreso orientado y coordinación con el consultorio periférico correspondiente.

10. Al egreso todo recién nacido tiene carnet de alta con los antecedentes del parto, vacunas e indicaciones.

11. El médico o enfermera dan indicaciones por escrito. 5

12. El servicio envia una copia de la epicrisis al Consultorio o Centro de Salud al que pertenece el niño. 10

13. Cuenta con servicio de laboratorio las 24 horas. 5

14. Cuenta con servicio de $\mathrm{Rx}$ las 24 horas. 5

VI - SUMINISTROS

1. Dispone de los medicamentos esenciales segun normas.

2. Dispone de material de enfermería (jeringa, alcohol, gasa, esparadrapo, sondas, etc.).

3. Tiene provisión regular de ropa limpia o desechable. 5

4. Hay elementos desechables para secarse las manos. 5

5. Dispone de material de limpieza (escobas, jabón, antisépticos).

6. Dispone de material de secretaría (papel, lápiz, etc.).

7. Dispone de repuestos para mantención y reparación de los aparatos (respiradores, mecánicos, incubadoras, etc.). 
8. Dispone de material educativo.

9. Existe material de colecta para exámenes bacteriológicos, hematológicos, etc.

10. En los últimos tres meses estos suministros han estado siempre disponibles.

\section{VII - EDUCACIÓN PARA LA SALUD}

1. Se efectúa educación a las puérperas.

2. Se realizan actividades de educación en cada visita a la madre y a su hijo en el alojamiento conjunto.

. Se estimula la participación de los padres en el cuidado del recién nacido patológico.

4. Se hace entrenamiento de las madres en prealta de R. N. patológico y de bajo peso.

5. Se hace promoción de la lactancia materna.

6. Se instruye a las madres sobre estimulación psicosocial del niño.

7. Se instruye a las madres sobre la importancia del control periódico de Crecimiento y Desarrolo.

8. Se provee un camet sanitario a cada niño egresado.

\section{ANEXO No 2 \\ ASPECTOS REFERIDOS A LA RELACION MADRE-HIJO APEGO Y ASPECTOS PSICOLOGICOS DE LA ATENCIÓN NEONATAL}

1. Se hace Educación Sanitaria a las puérperas.

2. Se dan contenidos de Lactancia Materna.

3. Se hace "rooming-in".

4. El R. N. se pone en contacto con la madre inemediatamente al nacer.

5. Se permite la presencia del padre en Sala de Dilatación

6. Se permite la presencia del padre en Sala de Expulsión.

7. Se permiten y estimulan visitas del cónyge a cualquier hora.

8. Se permite y estimula la presenciade otros hijos de la pareja.

9. Se ha suprimido el uso de biberones o mamaderas.

10. Se ha implementado un Banco de Leche Materna o mecanismo similar.

11. Se entrena a las madres en el manejo del R. N. de bajo peso.

12. Se permite y facilita el acceso de ambos padres a las

Unidades de Cuidado de Alta Riesgo.

Cadernos de Saúde Pública, R.J., 4(3): 426-443, out/dez, 1987. 